\section{Of mice and men}

W e would like to compliment, and complement, the recent Opinion in EMBO reports by Stuart Peirson and Russell Foster (2011), which calls attention to the potential obstacles associated with linking observations on light and circadian rhythmicity made on nocturnal mice to diurnally active humans. Pitfalls to consider include that qualitative extrapolations from short-lived rodents to long-lived humans, quantitative extrapolations of very different doses (Gold et al, 1992), and the varying sensitivities of each species to experimental optical radiation as a circadian stimulus (Bullough et al, 2006) can all have a critical influence on an experiment. Thus, Peirson \& Foster remind us that "humans are not big mice". We certainly agree, but we also thought it worthwhile to point out that human studies have their own interesting pitfalls, of which all researchers should be mindful.

Many investigations with humans-such as testing the effects of different light exposures on alertness, cognitive performance, well-being and depression - can suffer from what has been coined as the 'Hawthorne effect' ${ }^{\prime}$. The term is derived from a series of studies conducted at the Western Electric Company's Hawthorne Works near Chicago, Illinois, between 1924 and 1932, to test whether the productivity of workers would change with changing illumination levels. One important punch line was that productivity increased with almost any change that was made at the workplaces. One prevailing interpretation of these findings is that humans who know that they are being studied-and in most investigations they cannot help but notice-might exhibit responses that have little or nothing to do with what was intended as the experiment. Those who conduct circadian biology studies in humans try hard to eliminate possible 'Hawthorne effects', but every so often, all they can do is to hope for the best and expect the Hawthorne effect to be insignificant.

Even so, and despite the obstacles to circadian experiments with both mice and humans, the wealth of information from work in both species is indispensable. To exemplify, in the last handful of years alone, experimental research in mice has substantially contributed to our understanding of the retinal interface between visible light and circadian circuitry (Chen et al, 2011); has shown that disturbances of the circadian systems through manipulations of the light-dark cycles might accelerate carcinogenesis (Filipski et al, 2009); and has suggested that perinatal light exposure-through an imprinting of the stability of circadian systems (Ciarleglio et al, 2011) — might be related to a human's susceptibility to mood disorders (Erren et al, 2011a) and internal cancer developments later in life (Erren et al, 2011b). Future studies in humans must now examine whether, and to what extent, what was found in mice is applicable to and relevant for humans.

The bottom line is that we must be aware of, and first and foremost exploit, evolutionary legacies, such as the seemingly ubiquitous photoreceptive clockwork that marine and terrestrial vertebrates_-including mammals such as mice and humans-share (Erren et al, 2008). Translating insights from studies in animals to humans (Erren et al, $2011 a, b)$, and vice versa, into testable research can be a means to one end: to arrive at sensible answers to pressing questions about light and circadian clockworks that, no doubt, play key roles in human health and disease. Pitfalls, however, abound on either side, and we agree with Peirson \& Foster that they have to be recognized and monitored.

\section{CONFLICT OF INTEREST}

The authors declare that they have no conflict of interest.

\section{REFERENCES}

Bullough JD, Rea MS, Figueiro MG (2006) Cancer Causes Control 17: 375-383

Chen SK, Badea TC, Hattar S (2011) Nature 476: 92-95

Ciarleglio CM, Axley JC, Strauss BR, Gamble KL, McMahon DGA (2011) Nat Neurosci 14: 25-27 Erren TC, Nise MS, Meyer-Rochow VB (2011a) Psychopharmacology 216: 147-148

Erren TC, Groß JV, Meyer-Rochow VB (2011b) Chronobiol Int 28: 471-473

Erren TC, Erren M, Lerchi A, Meyer-Rochow VB (2008) Naturwissenschaften 95: 273-279

Filipski E, Subramanian P, Carrière J, Guettier C, Barbason H, Lévi F (2009) Mutat Res 680: 95-105 Gold LS, Manley NB, Ames BN (1992) Risk Anal 12: 579-588

Peirson SN, Foster RG (2011) EMBO Rep 12: 380

Thomas C. Erren is Professor of Occupational and Environmental Medicine and Director of the Institute and Policlinic for Occupational Medicine, Environmental Medicine and Prevention Research at the University of Cologne, Germany.

E-mail:tim.erren@uni-koeln.de

J. Valérie Groß and Melissa S. Koch are research scientists also at the Institute and Policlinic for

Occupational Medicine, Environmental Medicine and Prevention Research.

V. Benno Meyer-Rochow is Professor of Biology at the School of Engineering and Science, Jacobs University Bremen, Germany, and docent in the Departments of Biology and Physiology of the University of Oulu, Finland.

Published online 16 September 2011

EMBO reports (2011) 12, 991. doi:10.1038/embor.2011.185 